\title{
Characterization of single yeast cell phenotypes using microfluidic impedance cytometry and optical imaging
}

Niels Haandbæk ${ }^{* a}$, Sebastian C. Bürgel ${ }^{\mathrm{a}}$, Fabian Rudolf ${ }^{\mathrm{a}}$, Flavio Heer ${ }^{\mathrm{b}}$ and Andreas Hierlemann ${ }^{\mathrm{a}}$

${ }^{a}$ Dept. of Biosystems Science and Engineering, ETH Zurich, Basle, Switzerland

${ }^{\mathrm{b}}$ Zurich Instruments AG, Technoparkstrasse 1, 8005, Zurich, Switzerland

\section{Supporting information}

\section{Table of contents}

Table of contents $\quad$ S-1

1 Micrographs of the microfluidic device S-2

2 Finite element model S-2

3 Correlation between measured impedance and optical volume S-4

4 Principal component analysis of the measured impedance data S-6

5 Impedance events at the four measurement frequencies after classification S-7

6 Determining dielectric properties from experimental data using a model fitting approach S-8

7 Image processing algorithm $\quad S-10$

7.1 Define region-of-interest $S-11$

7.2 Update background
7.3

7.3 Move existing objects
$7.4-11$

7.4 Detect objects in current frame $S-12$

7.5 Match detected objects with moved objects $S-12$

7.6 Aging of unmatched objects $S-12$

7.7 Update matched objects with new positions $S-12$

$\begin{array}{ll}7.8 \text { Merge lists } & S-12\end{array}$

7.9 Kalman filter
$S-12$

8 Cells with multiple buds $\quad$ S-14

9 References $\quad$ S-14 


\section{Micrographs of the microfluidic device}

A

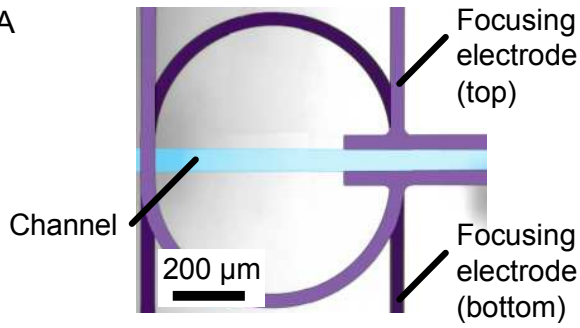

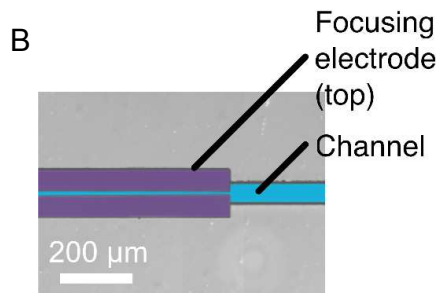

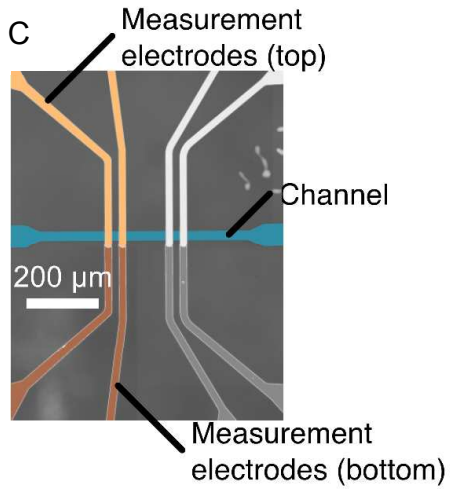

Figure S-1: Micrographs of the microfluidic device. (A) Micrograph of the initial part of the focusing region. The electrodes in the top layer of the device are routed from the top (lighter color), the bottom electrodes from the bottom (darker color). Particles enter from the left and exit on the right. (B) Micrograph of the end of the focusing region. Only the focusing electrodes on the top layer are visible. (C) Micrograph of the measurement region with two sets of measurement electrodes. Only the left (brown) pair is used in this work. The width of the channel is $60 \mu \mathrm{m}$ in the focusing region and $25 \mu \mathrm{m}$ in the measurement region. The focusing electrodes have a width of $40 \mu \mathrm{m}$ at the start of the focusing region, where they are aligned with the channel wall, and a width of $65 \mu \mathrm{m}$ at the end of the focusing region. The measurement electrodes are $18 \mu \mathrm{m}$ wide with a center-to-center spacing of $36 \mu \mathrm{m}$. The channel and electrodes have been colored for clarification.

\section{Finite element model}

A 3D model of the microfluidic channel was created and used for confirming the volume dependence of the impedance signal using the finite-element method (FEM). The geometry of the model is shown in Figure S-2. The simulations were performed by using the predefined electric current interface of the AC/DC module of COMSOL Multiphysics 4.3a (COMSOL AB, Sweden). The boundary conditions were set as insulating, except for the stimulation electrode, where a $1 \mathrm{~V}$ AC signal was applied.

The dielectric properties of the cells were taken from the work of Asami et al. ${ }^{1}$ As shown in the same work, the conductivity of the cell wall is directly proportional to the conductivity of the suspending medium. The proportionality factor for a medium with conductivity of $0.12 \mathrm{~S} / \mathrm{m}$ is approximately 0.25 . Therefore, the wall conductivity is defined as $0.03 \mathrm{~S} / \mathrm{m}$.

As demonstrated by Arnold et al., the conductivity of latex particles can be described by using two terms representing conductivity through the particle and conductivity around the particle as

$$
\sigma_{b}=\sigma_{b u l k}+2 \frac{K_{s}}{R}
$$

where $R$ is the radius of the particle, $\sigma_{\text {bulk }}$ is the bulk conductivity of the material and $K_{s}$ is the surface conductivity. ${ }^{2}$ The bulk conductivity is assumed to be effectively $0 \mathrm{~S} / \mathrm{m}$ and the surface conductivity is $1.2 \mathrm{nS} .^{3}$ 

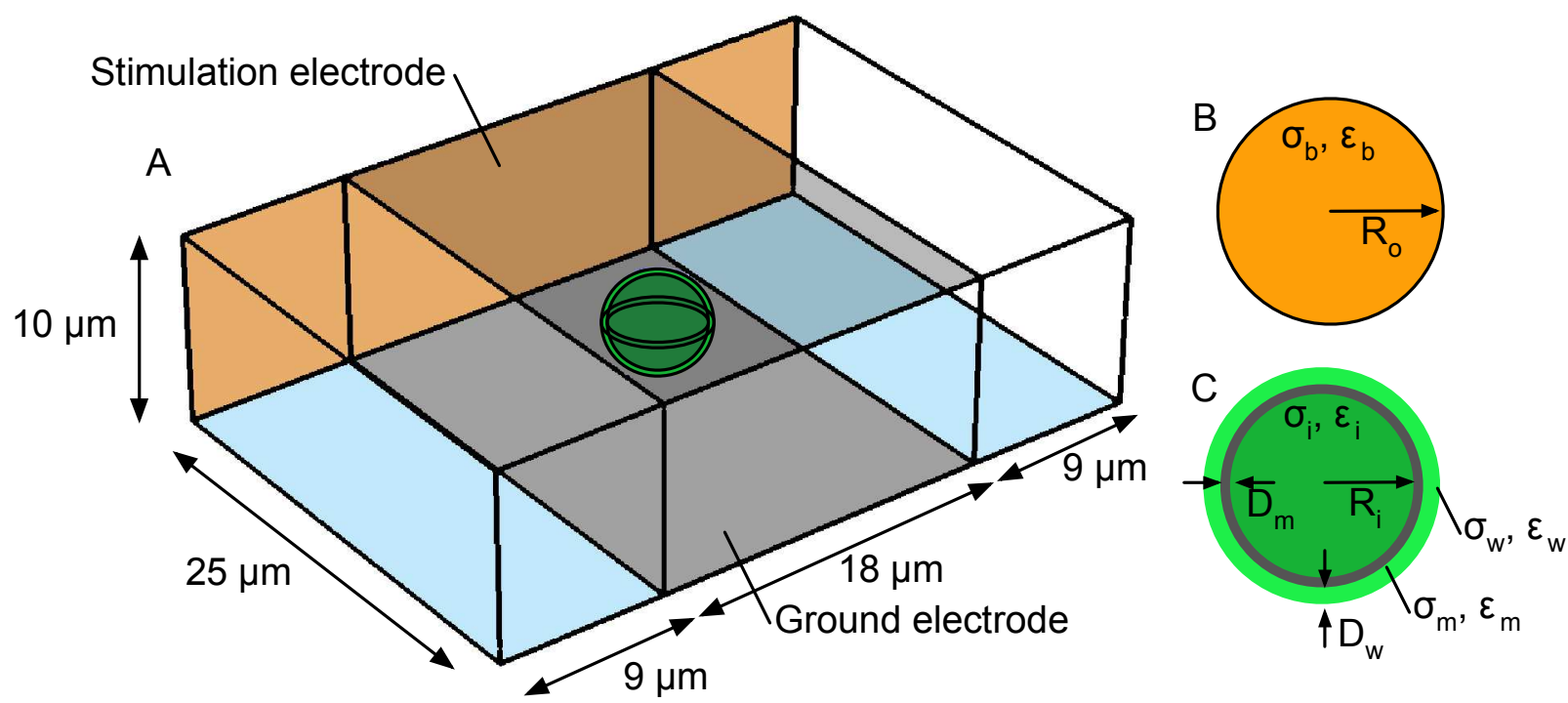

Figure S-2: 3D model of the microfluidic channel used for FEM simulations. (A) Geometry of the microfluidic channel. The dimensions are indicated in the figure. The object in the center of the channel models a bead, single cell or budding cell. The suspending medium in the channel is modeled with a conductivity of $0.12 \mathrm{~S} \mathrm{~m}^{-1}$ and dielectric constant of 80 . (B) The geometry of the bead consists of a sphere of radius $R_{o}$ with radius-dependent conductivity of $\sigma_{b}$ given by equation 4.1 , and dielectric constant, $\varepsilon_{b}$, of 2.6. (C) The geometry of the single cell consists of a sphere that represents the cytoplasm, covered by two further shells representing the cell membrane and the wall. The cytoplasm was modeled with dielectric properties of $\sigma_{i}=0.6 \mathrm{~S} / \mathrm{m}$ and $\varepsilon_{i}=58$. The cell membrane was modeled with a thickness, $D_{m}$, of $7 \mathrm{~nm}$, and dielectric properties of $\sigma_{m}=0 \mathrm{~S} / \mathrm{m}$ and $\varepsilon_{m}=5.2$. The cell wall was modeled with a thickness, $D_{w}$, of $250 \mathrm{~nm}$, and dielectric properties of $\sigma_{w}=0.03 \mathrm{~S} / \mathrm{m}$ and $\varepsilon_{w}=60$.

A common problem when using FEM is that of determining a suitable mesh that faithfully captures the most important features of the geometry. The mesh can become very complicated, when the geometry contains features of vastly differing length scales, such as the cell membrane in relation to the volume of the cytoplasm, which greatly increases the computation time. Furthermore, the numerical results of the model may appear inconsistent, when parts of the geometry are changed even slightly, because the mesh will be regenerated with a different structure.

Here we make use of two techniques to circumvent these problems. First of all, the cell membrane is not modeled using an actual geometrical shell. Instead, the cell membrane is modeled using a contact impedance, which is a thin layer approximation, where it is assumed that the current through the structure is oriented strictly in the normal direction. The approximation is valid, when the conductivity of the thin layer is much smaller than that of the surrounding medium. The approximation holds true for the presented experiments, because the conductivity of the membrane is at least six orders of magnitude smaller than that of the liquid. ${ }^{4}$ Secondly, we make use of the fact that the actual measurement is differential in nature. Every simulation is repeated twice using the same geometry and mesh, but with different material properties. In one simulation, the bead or cell is modeled using the actual dielectric properties, thereby simulating the case where an object is present in the measurement region. In the other simulation the dielectric properties are replaced with those of the surrounding medium, thereby simulating an empty measurement region. The results of the two simulations are then subtracted to generate the final results. As a consequence of applying these techniques, the simulations generate very robust results regardless of size or position of the cell within the channel.

Figure S-3 shows the volume dependence of the magnitude of the differential impedance signal for beads and cells at the low frequency used for the experiments. The budding cells were simulated with three different radii of the mother cells of $1.5 \mu \mathrm{m}$, $2.0 \mu \mathrm{m}$ and $2.5 \mu \mathrm{m}$, and a range of radii of the daughter cells between $1.0 \mu \mathrm{m}$ and $2.5 \mu \mathrm{m}$. As can be seen from the figure, there is a linear relationship between volume and impedance magnitude for beads, SCs and HBCs. In the case of the VBCs, the impedance magnitude stays relatively constant until the daughter cell reaches a size comparable to that of the mother cell. Afterwards, the impedance magnitude increases with total volume, as expected. The explanation is that the larger mother cell can shield a smaller daughter cell from the measurement current, thereby making the presence of the daughter cell invisible at the low measurement frequency. The figure also shows the result of a linear regression on the data. Comparing the slopes of the lines associated with the HBCs and VBCs, it can be seen that the magnitude of the differential impedance of the VBCs is only $75 \%$ that of the HBCs for a given volume. 


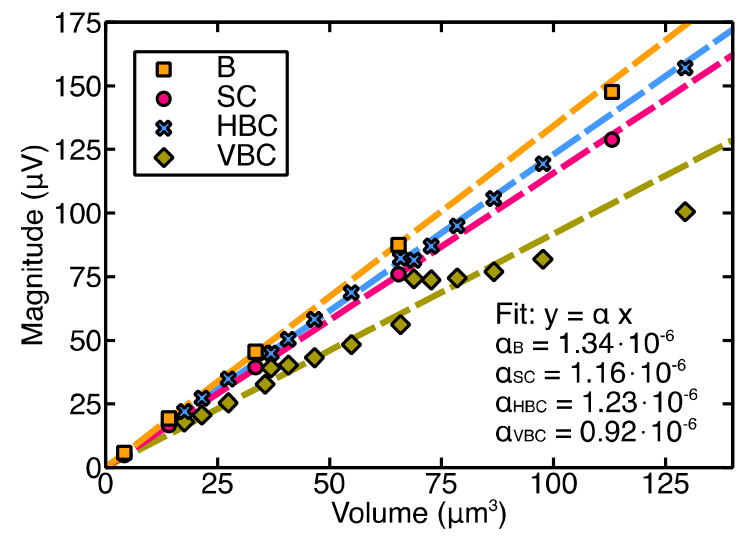

Figure S-3: Magnitude of the differential impedance signal for beads (B), single cells (SC), horizontally budding cells $(H B C)$ and vertically budding cells $(H B C)$, simulated for a range of cell volumes at a frequency of $0.55 \mathrm{MHz}$. For the budding cells, different combinations of daughter and mother volumes were simulated. The dashed lines are the result of a linear regression on the data using the formula indicated in the figure. The slopes of the resulting lines are also shown.

Figure S-4 shows the simulated opacity magnitude of the different cell morphologies over the frequency used for the experiment. The opacity was calculated using the simulated impedance magnitude at $0.55 \mathrm{MHz}$ as a reference, which is similar to what was done for the actual experimental results. As can be seen from the figure, the opacity of the VBCs is generally larger than that of both SCs and HBCs. Comparing Figure S-4A and Figure S-4B, it is evident that the opacity magnitude at higher frequencies is highly dependent on the cytoplasmic conductivity.
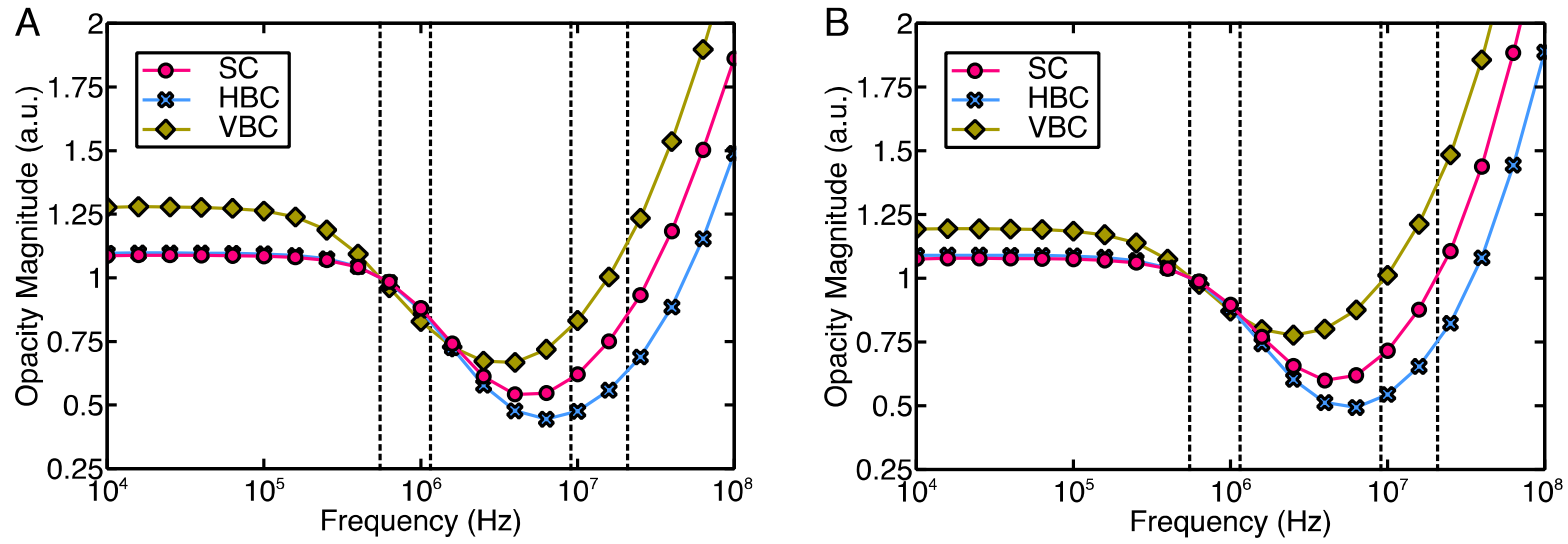

Figure S-4: Opacity magnitude of the different cell morphologies simulated over a frequency range from $10 \mathrm{kHz}$ to $100 \mathrm{MHz}$. The vertical dotted lines mark the four frequencies used for the experiments. The volume fraction, i.e., the ratio of total cell volume to volume of the measurement region, was kept at a value of $1.5 \%$ for both, the single-cell and the budding-cell simulations. The simulation was made by using the default value for cytoplasmic conductivity of $0.6 \mathrm{~S} \mathrm{~m} \mathrm{~m}^{-1}$ (A), but also using a value of $0.8 \mathrm{~S} \mathrm{~m}^{-1}(B)$, which was found by fitting a multi-shell model to the experimental results as described in the section "Determining dielectric properties from experimental data using a model fitting approach".

\section{Correlation between measured impedance and optical volume}

Figure S-6 shows the correlation between the volumes of the cells measured from the impedance signal at $0.55 \mathrm{MHz}$, and similar values determined by optical means. The optical volume was computed from the acquired image sequence of each cell using the procedure outlined in Figure S-5. First, the outline of each cell was detected. Next, the outline was divided into single-pixel-wide slices. Each slice was assumed to be a cylinder of single-pixel height and a diameter given by the length of the slice. The total volume was then computed by summing the volumes of each single-pixel slice cylinder. Unfortunately, the method does not allow the volume of the daughter cells of the VBCs to be explicitly extracted. As illustrated in Figure S-5B and C, the daughter cell will often overlap the mother cell. Therefore, the outline of the VBC will only be increased slightly in size in comparison to an SC of similar diameter. As a consequence, the volume of the VBCs determined optically is expected to only be slightly larger than that of the SCs. Similarly, as the HBCs are often not perfectly horizontal in the channel, the mother cell may overlap the daughter cell slightly, leading to a decrease in the size of the outline. Therefore, the optically determined volume of the HBCs is expected to be slightly smaller than the true volume. 

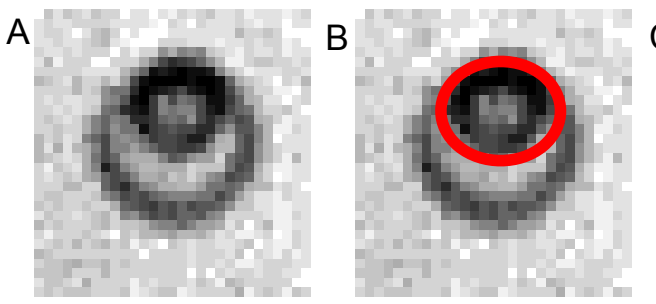

C

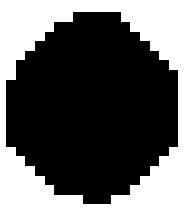

D

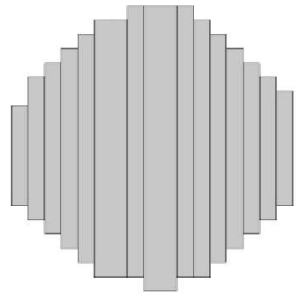

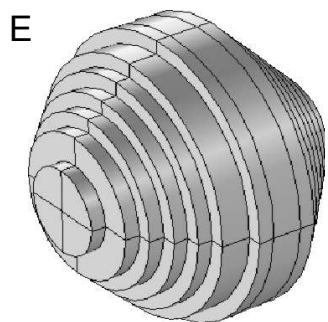

Figure S-5: Illustration of the algorithm for computing the volume from the acquired images. (A) Example of a VBC. (B) Daughter cell marked. (C) Outline of the complete cell, including daughter cell. (D) Outline divided into single-pixel wide slices. (E) Total volume is computed by treating each slice as a small cylinder.

The lines in Figure S-6 are the result of a simple linear regression on the data using a function of the form $y=\alpha x$. As can be seen in the figure, the correlation is quite good for both SCs $(\alpha=0.96)$ and HBCs $(\alpha=1.01)$, but not for VBCs $(\alpha=1.20)$. The reason for the lack of correlation for the VBCs is that cells that pass the electrodes in the vertical configuration obstruct much less of the electrode area in relation to their actual cell-volume in comparison to the SCs and HBCs.
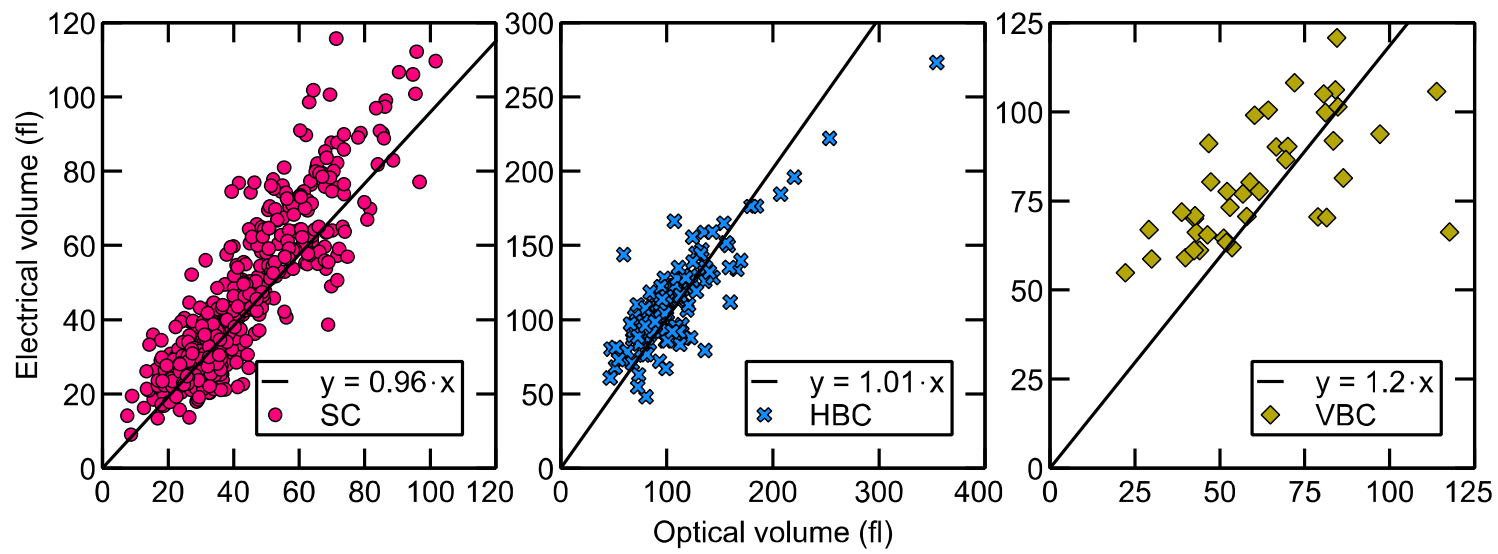

Figure S-6: Correlation between optically measured and electrically measured volume. $R^{2}(S C)=0.71, R^{2}(H B C)=0.71$, $R^{2}(V B C)=0.39$.

The mean and standard deviation of the optically determined volume of the different classes is summarized in Table S- 1 .

Table S-1: Mean and standard deviation of the volume of the beads (B), single daughter cells (SC,D), single mother cells $(S C, M)$, horizontally budding cells $(H B C)$ and vertically budding cells $(V B C)$, as determined optically.

\begin{tabular}{cccccc}
\hline & B & Class & & HBC & VBC \\
\hline Volume $\left(\mu \mathrm{m}^{3}\right)$ & $16.1 \pm 0.6$ & $29.5 \pm 7.3$ & $53.5 \pm 16.7$ & $105.3 \pm 39.4$ & $62.2 \pm 22.0$ \\
\hline
\end{tabular}

The optical images were further analyzed in order to evaluate the sizes of the two cells that make up a budding cell. The analysis involved fitting one or more circles to the outlines of the particles and cells as illustrated in Figure S-7A. By fitting two circles to the images of budding cells it was possible to the mother and daughter cells individually. Figure S-7B shows the resulting volume calculated by treating the detected circles as spheres of the same radius. In case of the cells, two bars are shown: one for the daughter cells labeled "X,D", and one for the mother cells labeled "X,M", where X is one of SC, HBC and VBC. As can be seen from the figure, the daughter cell volume of both single cells (SC,D) and budding cells (HBC,D and VBC,D) is similar. In contrast, the mean volume of the mother cells within a budding cell pair (HBC,M and VBC,M) is approximately $30 \%$ larger than the volume of the single mother cells (SC,M). 

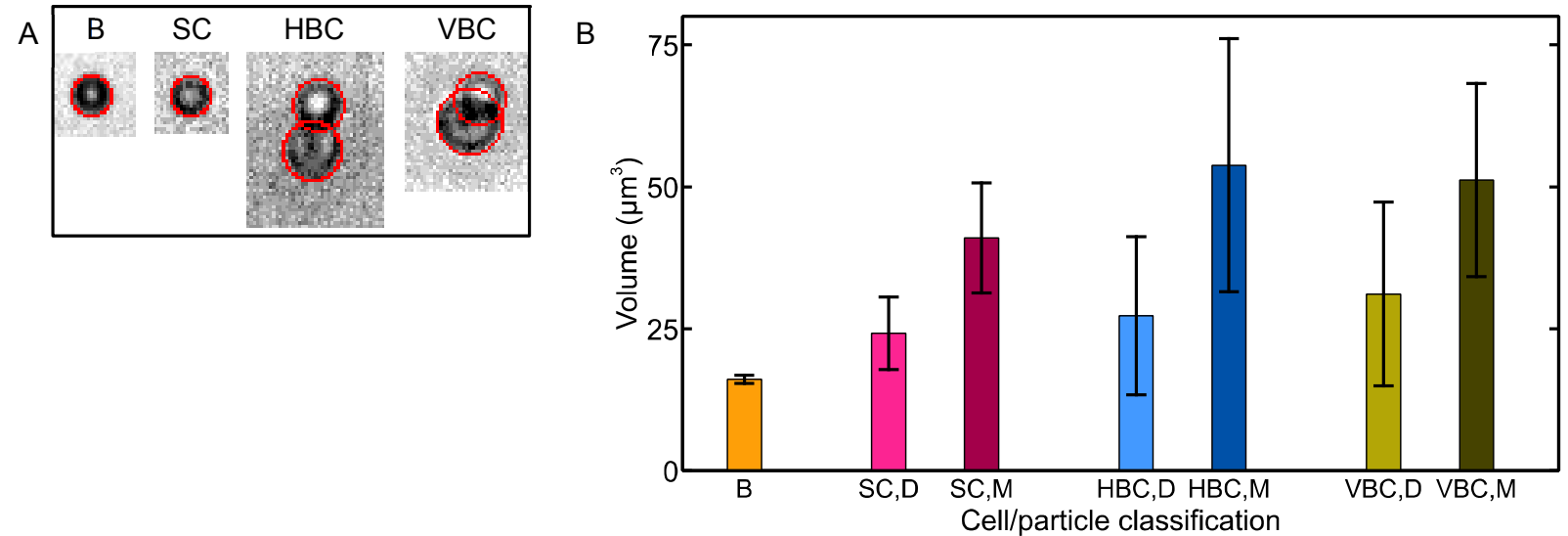

Figure S-7: (A) Examples of fitting circles to the optical images of beads (B), single cells (SC), horizontally budding cells $(H B C)$ and vertically budding cells (VBC). (B) Volume of the different object classes as calculated by fitting circles to the optical images. In case of the HBCs and VBCs the daughter cells are taken to be the smaller of the two cells in the pair.

\section{Principal component analysis of the measured impedance data}

In essence, the PCA is a linear transformation of the data into a new coordinate system. The basis vectors of the new coordinate system, called principal components, are constructed such that the greatest variance of the data comes to lie along the first vector, the second greatest along the second vector, and so on. The PCA was performed on a matrix $\boldsymbol{M}$ of the form

$$
\boldsymbol{M}=\left[\begin{array}{c}
\boldsymbol{m}_{1} \\
\vdots \\
\boldsymbol{m}_{N}
\end{array}\right],
$$

which consists of one row vector $\boldsymbol{m}_{n}$ for each measured cell or particle. The row vectors have the form

$$
\boldsymbol{m}_{n}=\left[\begin{array}{llllll}
v_{n} & x_{n}\left(f_{1}\right) & y_{n}\left(f_{1}\right) & \cdots & x_{n}\left(f_{4}\right) & y_{n}\left(f_{4}\right)
\end{array}\right],
$$

where $v_{n}$ is the velocity, $x_{n}\left(f_{i}\right)=\operatorname{Re}\left\{z_{n}\left(f_{i}\right)\right\}, y_{n}\left(f_{i}\right)=\operatorname{Im}\left\{z_{n}\left(f_{i}\right)\right\}$ and $z_{n}\left(f_{i}\right)$ is the complex peak-to-peak voltage of particle $n$ at frequency $f_{i}$. The result of the PCA is a new matrix $\boldsymbol{W}$ of the form

$$
\boldsymbol{W}=\left[\begin{array}{lll}
\boldsymbol{w}_{1}^{T} & \cdots & \boldsymbol{w}_{9}^{T}
\end{array}\right],
$$

which consists of nine column vectors of weights $\boldsymbol{w}_{k}=\left[w_{1}, \ldots, w_{9}\right]$ that, in total, map the rows of $\boldsymbol{M}$ to principal component scores:

$$
\begin{aligned}
& \boldsymbol{S}=\boldsymbol{M} \cdot \boldsymbol{W}=\left[\begin{array}{c}
\boldsymbol{s}_{1} \\
\vdots \\
\boldsymbol{s}_{N}
\end{array}\right], \\
& \boldsymbol{s}_{n}=\left[s_{n, 1}, \ldots, s_{n, 9}\right] .
\end{aligned}
$$

The first two principal components were found to account for more than $90 \%$ of the variability of the data set. Therefore, further analysis could be simplified to work with just the first two principal component scores instead of the original nine measured variables.

Finally, a classifier based on QDA was used to identify signatures in the impedance data for discriminating between the aforementioned classes. QDA is a supervised learning algorithm that can identify to which of a set of classes a new observation belongs, on the basis of a training set of observations whose class membership is known. ${ }^{5}$ After training, the algorithm can assign new observations to the most appropriate of the known classes. In this work, we used independent training and validation data sets. Impedance events that had been labeled based on the optical information were used as the training set and the resulting classes were those of "bead", "single cell" or "budding cell". The budding cells were further classified according to spatial orientation, i.e., the configuration in which they passed the measurement electrodes, as either horizontally budding cells or vertically budding cells. QDA assumes that the different classes are drawn from multivariate normal distributions with varying mean and covariance. The algorithm was found to adequately discriminate between the different classes, although the measured data do not entirely satisfy these criteria.

The volume of the cells may be determined from the magnitude of the impedance signal at $0.55 \mathrm{MHz}$ by using the monodisperse beads as a reference. The beads have a mean diameter specified by the manufacturer as $3.1 \pm 0.062 \mu \mathrm{m}$, which corresponds to a volume of $16 \pm 0.96 \mathrm{fl}$. The knowledge of the volume of the beads can be used to convert the measured impedance values of the cells into actual physical volumes as follows. Let $Z_{N, m}(0.55 \mathrm{MHz})$ be the measured complex impedance of a particle or cell $m$ of the class $N$ at the frequency of $0.55 \mathrm{MHz}$. The mean of the magnitude of the impedance of all the beads is then given by 


$$
\overline{\left|Z_{B}(0.55 M H z)\right|}=\frac{1}{M} \sum_{m=1}^{M}\left|Z_{B, m}(0.55 M H z)\right|,
$$

where $M$ is the total number of detected beads. The scaling factor $\alpha$ is then given by

$$
\alpha=\frac{4 \pi r_{B}{ }^{3}}{3 \overline{\left|Z_{B}(0.55 M H z)\right|}},
$$

where $r_{B}$ is the mean bead radius. Finally, the effective volume of any detected cell or particle can be computed using

$$
V_{N, m}=\alpha\left|Z_{N, m}(0.55 \mathrm{MHz})\right|
$$

Table S-2 shows the weights of the principal components associated with the measured impedance data.

Table S-2: Table of weights of the first two principal components. The weights are organized according to equation S-4.2. $X$ refers to the real part and $Y$ to the imaginary part of the measured impedance signal.

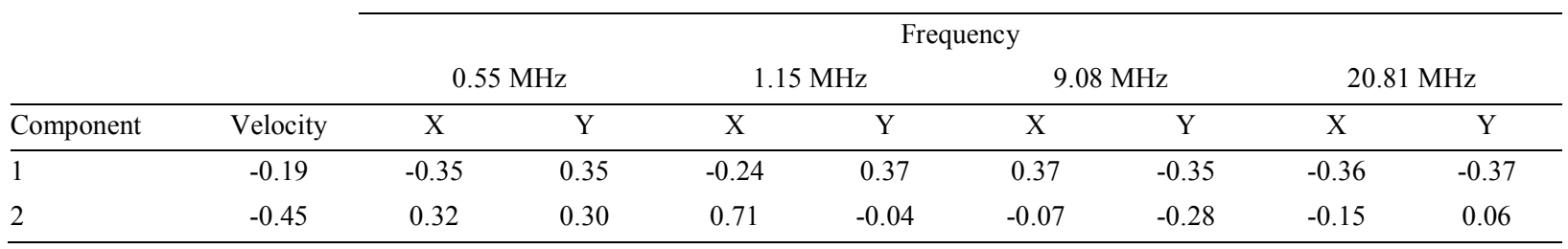

\section{Impedance events at the four measurement frequencies after classification}

Figure S-8 shows the complete results of the measurement after classification using the QDA algorithm. The SCs have further been classified as daughter (SC,D) or mother (SC,M) cells based on the magnitude of the impedance at $0.55 \mathrm{MHz}$.

The exact placement of the events within the complex plane depends on a number of factors related to the physical properties of the complete system. For example, even though great care has been taken during the design of the microfluidic device in order to ensure that the two pairs of measurement electrodes are as similar as possible, small differences in geometry are unavoidable because of the physical fabrication process. Differences in the surface properties of the electrodes may also lead to slight differences in the double layer capacitance that forms between the electrodes and the bulk of the suspending medium. Similarly, the measurement electronics have been designed by using high-precision passive and active components, but even then, small differences in gain between the two paths connected to the measurement electrodes cannot be avoided.

These differences may be small in absolute numbers. However, due to the differential measurement principle, even very small differences between the signals measured from the two pairs of electrodes will give rise to a detectable offset in the final results. Furthermore, owing to the lock-in measurement principle, which measures the magnitude and phase of the response from the microfluidic device in relation to a reference frequency, the signal delay through the system will also cause a frequencydependent phase shift. This effect is visible in the figure as an overall frequency-dependent rotation of the data.

As a consequence of all these effects, one should be careful when drawing conclusions about changes in dielectric properties from the raw measurement results. The plots of the raw data are only useful for investigating differences in impedance response between the different classes. 

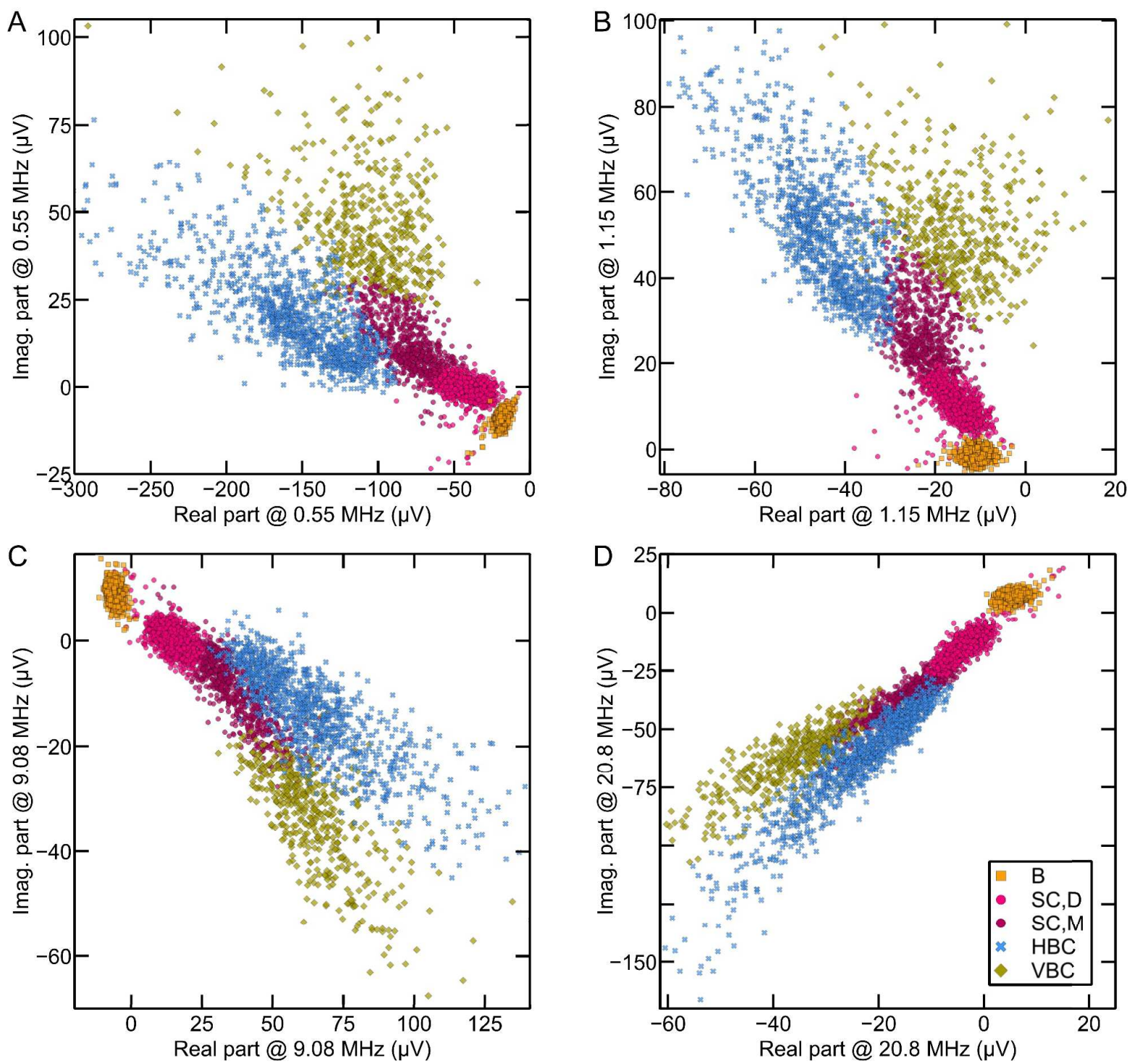

Figure S-8: Imaginary versus real part of the measured impedance of all events detected in the experiment at frequencies of (A) $0.55 \mathrm{MHz}$, (B) $1.15 \mathrm{MHz}$, (C) $9.08 \mathrm{MHz}$ and (D) $20.8 \mathrm{MHz}$. The symbols used for the different classes are the same for all plots.

\section{Determining dielectric properties from experimental data using a model fitting approach}

The current through the microfluidic channel is a function of the electrode geometry, suspension medium, as well as the dielectric properties of the cells and particles. The impedance spectroscope measures the currents passing between both pairs of electrodes simultaneously and then subtracts them. The resulting differential signal is, therefore, a measure of the contrast between the current through the empty channel, and the channel when a cell or particle is present. As a consequence of the differential measurement principle, it is not possible to directly convert the measured impedance signal into corresponding dielectric properties for the cells and particles. In order to measure the dielectric properties, it is necessary to have an absolute reference in addition to the differential signal.

One possibility would be to make a single-ended measurement of the empty channel in parallel to the differential measurement. However, such a measurement is currently not possible due to limitations in the measurement electronics. Another possibility, which was used here, is to apply a model-based approach, where a model of the channel with and without cell is matched to the measured data. As shown in previous work, ${ }^{6}$ it is possible to match a $3 \mathrm{D}$ FEM similar to the one shown in Figure S-2 to the measured data. The advantage of that method is that the model can represent arbitrary geometries, such as budding cells, without the need for developing a specific mathematical model of the system. The disadvantage is the very long computation time needed to evaluate the model. Another consideration is the many degrees of freedom that are associated with the 3D model, which results in a more detailed impedance spectrum being needed in order to properly constrain the curve fitting procedure. The limitations of the 3D FEM approach, combined with the impedance spectrum being measured at just four frequencies, motivated us to make use of a simpler model in the presented work. 
The model is based on the "single shell" model of Pauly and Schwan extended to handle multiple shells using the work of Irimajira et $a l .{ }^{7,8}$ The main innovation of the presented work is a method that enables the parameters of the model to be fitted to the results of a differential single-cell impedance measurement.

The model expresses the dielectric properties of a cell or particle in the microfluidic channel in terms of the complex permittivity defined as

$$
\varepsilon^{*}=\varepsilon+\frac{\sigma}{j \omega \varepsilon_{0}}
$$

where $\varepsilon$ is the relative permittivity, $\varepsilon_{0}$ the vacuum permittivity, $\sigma$ the conductivity, $\omega$ the radial frequency under consideration, and $j=\sqrt{-1}$. The frequency dependence of the complex permittivity is implicitly assumed in the following equations. The geometry of the model is shown in the following figure. The structure of the model is similar to that used for the 3D FEM.
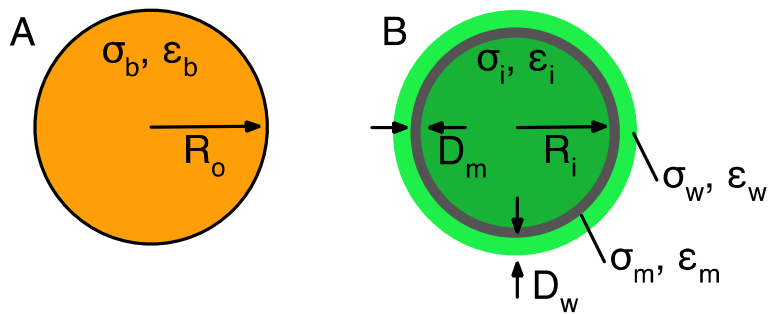

Figure S-9: Geometry of a bead (A) and a cell (B) used in the electrical model. The outer radius, $R_{o}$, defines the radius of the complete cell or particle. The inner radius, $R_{i}$, defines the radius of the volume associated with the cytoplasm of the cell. $D_{m}$ and $D_{w}$ define the thickness of the shells associated with the cell membrane and cell wall, respectively.

The complex permittivity of the bead is given by equation 6.1 . The complex permittivity of the cell is defined recursively. ${ }^{8}$ First, the complex permittivity of the cytoplasm covered by the cell membrane is defined as

where

$$
\varepsilon_{p}^{*}=\varepsilon_{m}^{*} \frac{2\left(1-v_{1}\right) \varepsilon_{m}^{*}+\left(1+2 v_{1}\right) \varepsilon_{i}^{*}}{\left(2+v_{1}\right) \varepsilon_{m}^{*}+\left(1-v_{1}\right) \varepsilon_{i}^{*}}
$$

$$
v_{1}=\left(1-D_{m} / R_{i}\right)^{3}
$$

Next, the complex permittivity of the entire cell may be defined as

$$
\varepsilon_{c}^{*}=\varepsilon_{w}^{*} \frac{2\left(1-v_{2}\right) \varepsilon_{w}^{*}+\left(1+2 v_{2}\right) \varepsilon_{p}^{*}}{\left(2+v_{2}\right) \varepsilon_{w}^{*}+\left(1-v_{2}\right) \varepsilon_{p}^{*}}
$$

where

$$
v_{2}=\left(1-D_{w} / R_{o}\right)^{3}
$$

Finally, the complex permittivity of the cell (or particle) within the region between the measurement electrodes is determined using the Clausius-Mossotti relation of

$$
\Phi_{C M}=\frac{\varepsilon_{c}^{*}-\varepsilon_{o}^{*}}{\varepsilon_{c}^{*}+2 \varepsilon_{o}^{*}}
$$

combined with Maxwell mixture theory as

$$
\varepsilon_{\text {total }}^{*}=\varepsilon_{o}^{*} \frac{1+2 \mathrm{~V} \Phi_{C M}}{1-V \Phi_{C M}}
$$

The volume fraction, $V$, in equation 6.7 is given by

$$
V=\frac{4 / 3 \pi R_{o}{ }^{3}}{w d h}
$$

where $w$ is the electrode width, $h$ the channel height, and $d$ the channel width. The volume fraction of the cells and particles used in this work is typically below $2 \%$. Therefore, it is not necessary to use the geometry factor derived by Gawad et al. ${ }^{9}$ to adjust the volume fraction. The frequency-dependent impedance of the microfluidic channel may now be calculated as

$$
Z(f)=\left(\frac{j 2 \pi f \varepsilon_{\text {total }}^{*} w d}{h}\right)^{-1}
$$


The final piece of the puzzle is to define a method of normalization that would allow for comparing the measured impedance values to those computed by the model. We first ensure that the dielectric properties are determined in a size-independent manner by considering the opacity given by

$$
o_{N, m}\left(f_{i}\right)=\frac{Z_{N, m}\left(f_{i}\right)}{Z_{N, m}\left(f_{1}\right)}
$$

where $Z_{N, m}\left(f_{i}\right)$ is the complex impedance at one of the four frequencies $f_{l}$ to $f_{4}$, and $o_{N, m}\left(f_{i}\right)$ is the resulting opacity. The subscript $N$ refers to the class of the object, and $m$ is an index denoting one of the measured cells or particles. We then make use of the fact that the impedance of the beads is very well defined, due to their uniform composition and well-characterized material, by normalizing the opacity of the cells with the opacity of the beads. The normalization is performed on the opacity value of each detected cell, at each of the four measurement frequencies, using

$$
Q_{N, m}\left(f_{i}\right)=\frac{O_{N, m}\left(f_{i}\right)}{\left|O_{B}\left(f_{i}\right)\right|}
$$

In equation $6.11,\left|O_{B}\left(f_{i}\right)\right|$ is the mean of the opacity of the beads at frequency $f_{i}$. Similar normalized opacity values are calculated using the simulation model, with the only exception being that the opacity of the beads is immediately defined.

A curve fitting procedure was then used in order to estimate values for the dielectric properties of the cells. The curve fitting procedure gradually adjusts the dielectric properties of the simulation model in order to minimize the mean square error between the measured and simulated normalized opacity values at the four frequencies. The degrees of freedom of the model were limited to the smallest possible set that would still give a satisfactory quality of fit $\left(\mathrm{R}^{2}>0.99\right)$.

Table S-3 shows the result of fitting the simulation model to the mean values of the normalized opacity data measured for the different cell classes. The ranges in the table are the maximum and minimum values of the properties found during 100 runs of the curve fitting procedure executed with randomized starting values.

Table S-3: Physical and dielectric properties of the different cell classes determined by fitting the model to the mean values of the measured impedance data.

\begin{tabular}{lccc}
\hline Species & $\begin{array}{c}\text { Cell wall thickness } \\
(\boldsymbol{\mu m})\end{array}$ & $\begin{array}{c}\text { Cell wall conductivity } \\
\left(\mathbf{S} \cdot \mathbf{m}^{-\mathbf{1}}\right)\end{array}$ & $\begin{array}{c}\text { Cytoplasm conductivity } \\
\left(\mathbf{S} \cdot \mathbf{m}^{\mathbf{- 1}}\right)\end{array}$ \\
\hline SC,D & $0.15-0.18$ & $0.017-0.019$ & $0.73-0.83$ \\
SC,M & $0.16-0.17$ & $0.017-0.019$ & $0.81-0.88$ \\
HBC & $0.21-0.24$ & $0.023-0.026$ & $0.79-0.92$ \\
VBC & $0.13-0.14$ & $0.014-0.015$ & $2.5-2.7$ \\
\hline
\end{tabular}

\section{Image processing algorithm}

The image processing algorithm used for detecting cells and particles, in the following referred to simply as "objects", is illustrated in the following figure. The different steps will be explained in more detail in the following sections. 


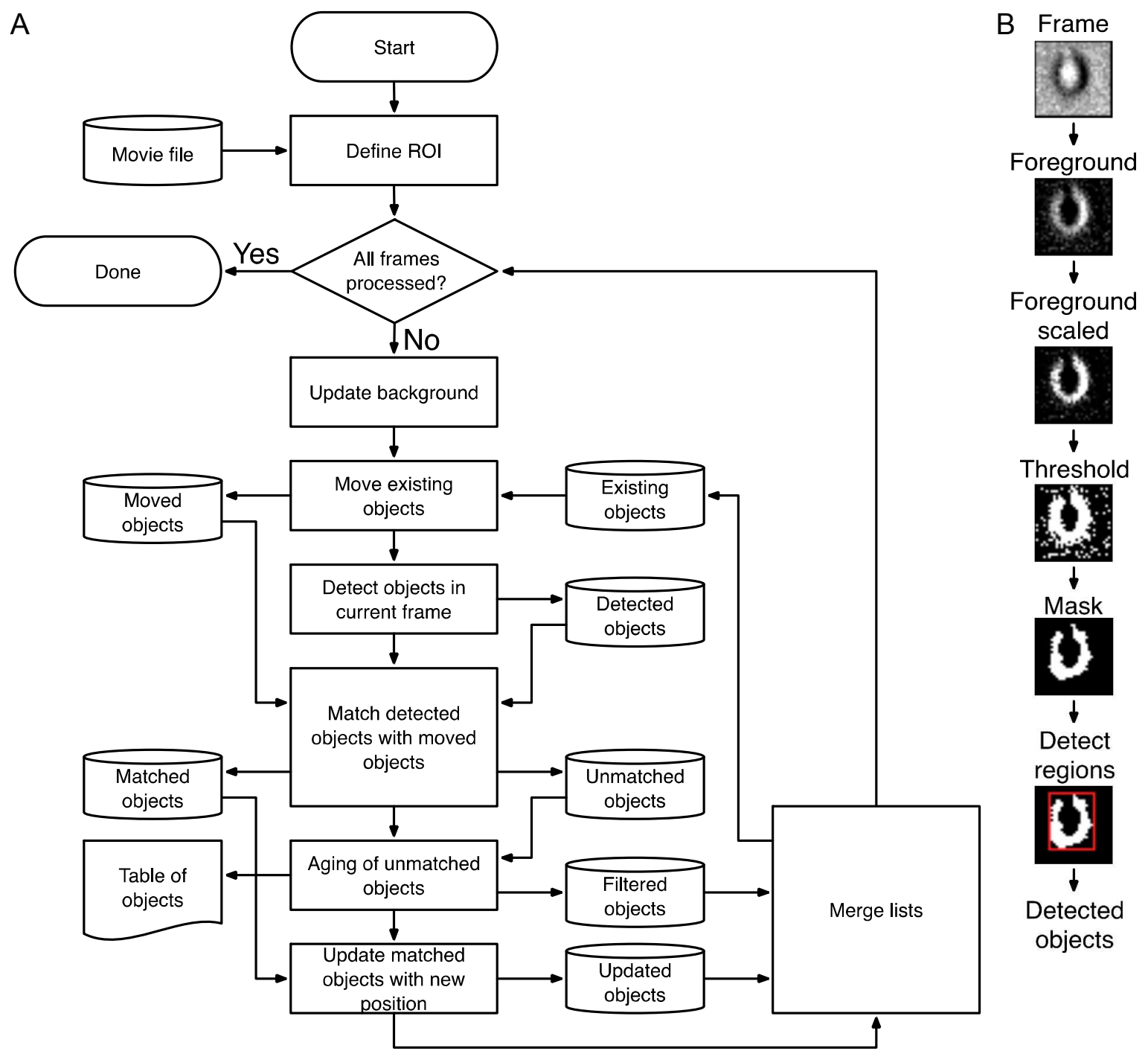

Figure S-10: Details of the image-processing algorithm. (A) Image-processing algorithm flow diagram. The algorithm takes the frame sequence recorded by the high-speed camera as input and produces a list of detected objects, which includes various extracted parameters such as the object area and velocity. The algorithm detects cells or particles that are visible in a given frame and tracks their frame-to-frame movement. (B) Details of the operations made on the images in order to detect objects.

\subsection{Define region-of-interest}

In this step the user may define the region-of-interest (ROI), which should be analyzed by the algorithm. The camera may have recorded a larger area of the microfluidic channel than what is strictly necessary for detecting the cells. The purpose of this step is to limit the area, and, therefore, the amount of data that needs to be analyzed to detect all cells.

\subsection{Update background}

Objects are detected by differentiating them from the background of the individual frames. The background is detected by averaging multiple frames by means of an exponential-moving-average filter. The filter works on a frame-by-frame basis. The background pixel, $B$, at location $(x, y)$ of frame $k$ is computed recursively as follows

$$
B_{x, y}[k]=\alpha B_{x, y}[k-1]+(1-\alpha) F_{x, y}[k],
$$

where $F_{x, y}$ is the pixel at location (x,y) of the current frame. The constant $\alpha$ defines the time-constant of the filter and is typically set to 0.9 .

\subsection{Move existing objects}

The algorithm must ensure that every object that passes through the ROI is only detected once. Therefore, it maintains a list of objects that are potentially visible. When a new frame is processed, the algorithm computes the expected positions of the already known objects within this frame by means of a Kalman filter. The filter implementation is explained in more detail in a following 
section. In essence, the expected position $\boldsymbol{p}_{n, k}$ of particle $n$ in frame $k$ is computed based on the last position of the object, $\boldsymbol{p}_{n, k-1}$, its velocity $\boldsymbol{v}_{n, k-1}$ at that time and the time increment $\Delta t$ using:

$$
\boldsymbol{p}_{n, k}=\boldsymbol{v}_{n, k-1} \Delta \mathrm{t}+\boldsymbol{p}_{n, k-1}
$$

$\boldsymbol{p}$ and $\boldsymbol{v}$ are both two-dimensional vectors and the time increment is set to unity for simplicity.

\subsection{Detect objects in current frame}

Figure S-10B illustrates the morphological operations made on the individual frames in order to detect objects. First, the image foreground is detected by subtracting the background as calculated using equation 6.1 from the current frame (Foreground). The result of the operation is that regions within the frame corresponding to cells or particles will become lighter than the surroundings. Squaring the pixel values further increases the contrast (Foreground scaled). The data is then converted from grayscale to binary (i.e. black-and-white) by means of a threshold operation (Threshold). All pixels with values above the threshold are set to ' 1 ' and all others to ' 0 '. Morphological image operations were used to remove lonely pixels, which correspond to noise, and to refine the boundary of large groups of adjacent pixels (Mask). ${ }^{10}$ Finally, regions of adjacent pixels with a cumulative area larger than 40 pixels $^{2}$ were detected and returned as objects (Detect regions) with positions given by the centroid of the bounding box around the region as shown by the red box.

\subsection{Match detected objects with moved objects}

The positions of the objects detected in the current frame are then matched to the expected positions of the existing objects by means of Murty's algorithm. ${ }^{11}$ Essentially, the algorithm finds the optimal assignment such that the distance between expected and actual positions is minimized. The output of this step consists of two lists: A list of objects for which an existing object was found, and a list with the remaining new objects. The list of remaining objects represents new objects that have entered into the field of view since processing of the last frame, or objects for which the estimation of the movement is very different from the actual movement of the objects.

\subsection{Aging of unmatched objects}

In this step the age of the unmatched objects is incremented. All objects for which the age exceeds a specific threshold are removed from processing and added to the list of results. The remaining objects are added to another list for consideration in the next processing step. The effect of this step is that objects that have passed through the ROI and, therefore, can no longer be matched to objects within the ROI, are detected as such and added to the results. In order to increase the robustness of the algorithm and make it less sensitive to errors, the objects are not removed from processing immediately, but only after a certain number of frames have passed in which it has not been possible to detect a matching object within the ROI.

\subsection{Update matched objects with new positions}

At this step in the algorithm, the objects for which a new position has been detected, because they were detected in the current frame, will have their positions updated to the new values. The velocity of the objects will also be updated accordingly. The position and velocity of the objects are fed into the Kalman filter as explained in a following section.

\section{8 $\quad$ Merge lists}

The final step in the algorithm is to merge the lists of objects into a single list. The objects in the single list represent new objects that have never been seen before, i.e., objects that could not be matched with any known object within the ROI, objects that have potentially left the ROI, i.e., objects that could not be matched, but are also not old enough to be completely removed from future consideration, and existing objects that were found again in the current frame and, therefore, have had their positions and velocities updated.

\subsection{Kalman filter}

The Kalman filter estimates a process by using a form of feedback control: the filter estimates the process state at some time and then obtains feedback in the form of (noisy) measurements. As such, the equations for the Kalman filter fall into two groups: time update equations and measurement update equations. The time update equations are responsible for projecting forward (in time) the current state and error covariance estimates to obtain the a priori estimates for the next time step. The measurement update equations are responsible for the feedback, i.e. for incorporating a new measurement into the a priori estimate to obtain an improved a posteriori estimate.

The algorithm maintains state information for each detected object, a cell or a particle, in the form of its position and velocity. During the processing of a frame, the state of the already detected objects is estimated by means of a discrete Kalman filter. ${ }^{12}$ The Kalman filter provides a computationally efficient means to estimate the state of a process, in this case the object position and velocity, in a way that minimizes the mean of the squared error. The filter supports estimations of future object positions even though only approximate equations governing the movements of the objects are known.

The Kalman filter receives two inputs in the form of the measurement vector, $\boldsymbol{z}_{k}$, and control vector, $\boldsymbol{u}_{k}$. The measurement vector represents the measured position and velocity of a particle as determined from the image sequence using the above algorithm. The 
control vector represents a known external force on the cells and particles. In this case there is no external control, so $\boldsymbol{u}_{k}$ is simply zero.

The Kalman filter contains the state vector

$$
\boldsymbol{x}_{k}=\left[\begin{array}{llll}
p_{x} & v_{x} & p_{y} & v_{y}
\end{array}\right]^{T}
$$

where $p_{x}, p_{y}$ denote the position and $v_{x}, v_{y}$ denote the velocity of the object at frame $k$. The state vector has the same form as the measurement vector. The state transition matrix

$$
\boldsymbol{A}=\left[\begin{array}{llll}
1 & 1 & 0 & 0 \\
0 & 1 & 0 & 0 \\
0 & 0 & 1 & 1 \\
0 & 0 & 0 & 1
\end{array}\right]
$$

relates the state in the previous frame $k-1$ to the state in the current frame $k$ by means of the equation

$$
\boldsymbol{x}_{k}=\boldsymbol{A} \boldsymbol{x}_{k-1} \text {. }
$$

As can be seen from the form of $\boldsymbol{A}$, the position in the new state is simply computed by adding the velocity. The velocity in the new state is assumed to be the same as in the previous state.

The state error covariance matrix, which is initially given by

$$
\boldsymbol{P}=\left[\begin{array}{cccc}
10 & 0 & 0 & 0 \\
0 & 2 & 0 & 0 \\
0 & 0 & 10 & 0 \\
0 & 0 & 0 & 2
\end{array}\right],
$$

models the uncertainty in the estimate of the state. In essence, the initial value of $\boldsymbol{P}$ defines the variance in the estimate of $p_{x}$ and $p_{y}$ as 10 pixels, and the variance in the estimate of $\mathrm{v}_{\mathrm{x}}$ and $\mathrm{v}_{\mathrm{y}}$ as 2 pixels. Furthermore, the estimates of position and velocity are assumed to be uncorrelated as indicated by the off-diagonal elements in the matrix being zero.

The estimated process error covariance matrix models intrinsic uncertainty in the movement of the cells and particles. It is initially given by

$$
\boldsymbol{Q}=\left[\begin{array}{cccc}
10^{3} & 0 & 0 & 0 \\
0 & 1 & 0 & 0 \\
0 & 0 & 10^{2} & 0 \\
0 & 0 & 0 & 1
\end{array}\right]
$$

Similarly, the measurement covariance matrix models the uncertainty in the determination of the different state parameters. It is initially given by

$$
R=\left[\begin{array}{cccc}
10^{2} & 0 & 0 & 0 \\
0 & 10 & 0 & 0 \\
0 & 0 & 10^{2} & 0 \\
0 & 0 & 0 & 10
\end{array}\right]
$$

The overall algorithm is quite insensitive to the precise values used for $\boldsymbol{Q}$ and $\boldsymbol{R}$. The above values were simply found through trial-and-error.

Next, the observation matrix is given by

$$
\boldsymbol{H}=\left[\begin{array}{llll}
1 & 0 & 0 & 0 \\
0 & 1 & 0 & 0 \\
0 & 0 & 1 & 0 \\
0 & 0 & 0 & 1
\end{array}\right]
$$

The observation matrix is used to translate the state vector into a measurement vector. As the particle detection algorithm determines the absolute position and velocity of the particles, the matrix is simply the identity matrix.

The update equations of the filter are as follows. First, the state in the current frame is estimated based on the state in the previous frame using

$$
\hat{x}_{k}=A x_{k-1} \text {. }
$$

Next, the estimate of the error in the state is updated using 


$$
\widehat{\boldsymbol{P}}_{k}=\boldsymbol{A} \boldsymbol{P}_{k-1} \boldsymbol{A}^{T}+\boldsymbol{Q} .
$$

Then, a factor known as the Kalman gain is computed using

$$
\boldsymbol{K}=\widehat{\boldsymbol{P}}_{k} \boldsymbol{H}^{T}\left(\boldsymbol{H} \widehat{\boldsymbol{P}}_{k} \boldsymbol{H}^{T}+\boldsymbol{R}\right)^{-1} .
$$

The Kalman gain is a measure of how well the actual measurement input can be trusted. In case the measurement input is very noisy, the filter will assign a higher weight to the estimated value of the next state in comparison to the measured value. The Kalman gain is used in the actual computation of the next state using

$$
\boldsymbol{x}_{k}=\widehat{\boldsymbol{x}}_{k}+\boldsymbol{K}\left(\mathbf{z}_{k}-\boldsymbol{H} \widehat{\boldsymbol{x}}_{k}\right) \text {. }
$$

As seen from the equation, the next state is computed based on the estimate of the next state, $\widehat{\boldsymbol{x}}_{k}$, the actual measurement vector, $z_{k}$, and the Kalman gain. The estimate of the error in the state is updated using a similar equation given by

$$
\boldsymbol{P}_{k}=\widehat{\boldsymbol{P}}_{k}-\boldsymbol{K} \boldsymbol{H} \widehat{\boldsymbol{P}}_{k} .
$$

The final output of the Kalman filter is a more reliable estimate of the position of a cell or particle in a following frame based on information in previous frames. The use of the Kalman filter makes the entire algorithm robust in situations where, for example, the particle detection algorithm alone loses track of a particle.

\section{Cells with multiple buds}

Figure S-11 shows two examples of cells that have begun producing a new bud without having properly separated. The last panel in the figure shows an example of three budding cells sticking together.

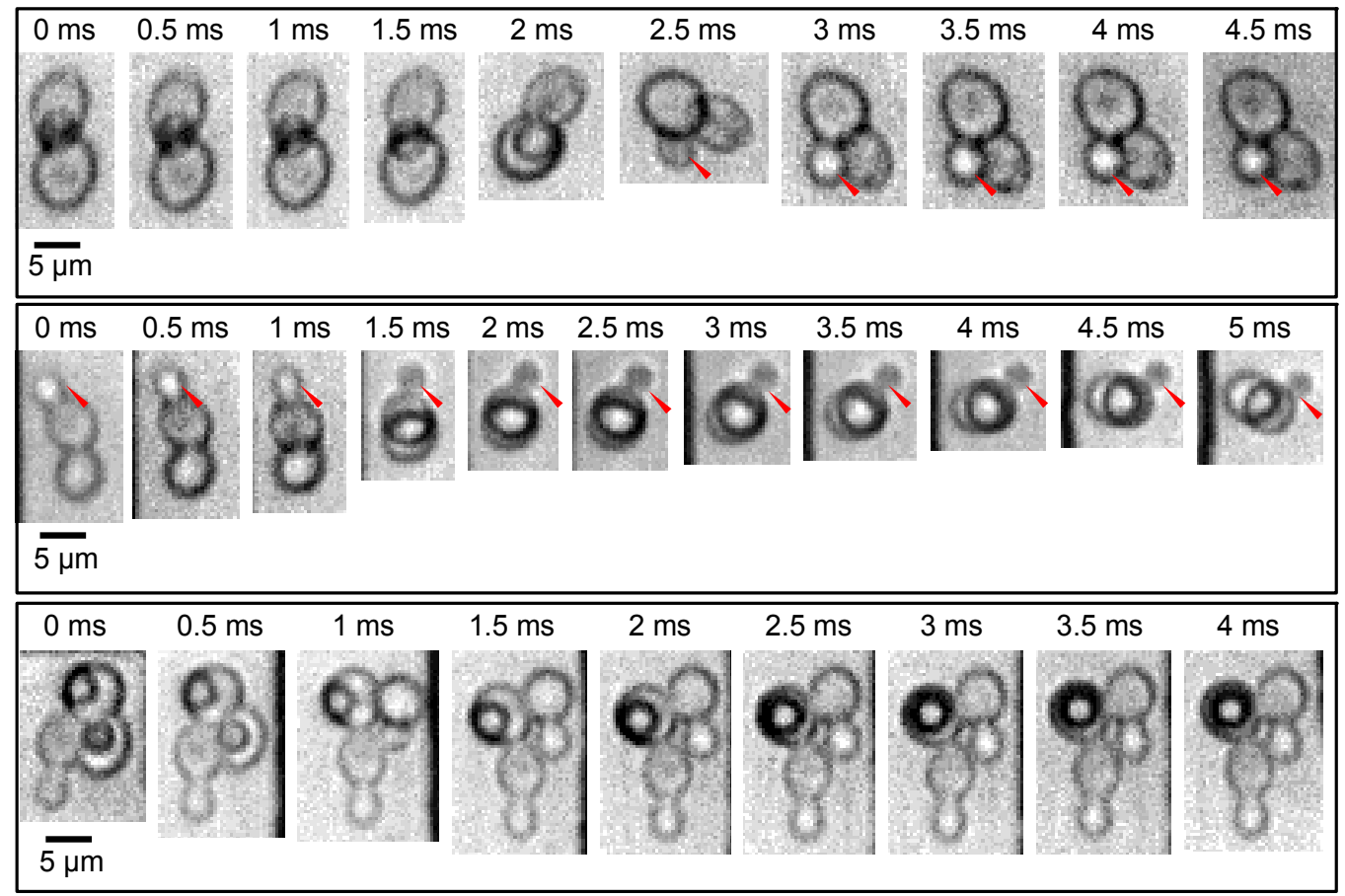

Figure S-11: Examples of cells with one or more extra buds and a larger conglomeration of cells. The red arrow shows the extra bud. Larger groupings of cells such as these occur by chance either because mother and daughter cell fail to separate properly after mitosis or simply because multiple cells stick together.

\section{References}

(1) Asami, K.; Yonezawa, T. Dielectric Behavior of Wild-Type Yeast and Vacuole-Deficient Mutant over a Frequency Range of $10 \mathrm{kHz}$ to $10 \mathrm{GHz}$. Biophys. J. 1996, 71 (4), 2192-2200.

(2) Arnold, W.; Zimmermann, U. ELECTRO-ROTATION: DEVELOPMENT OF A TECHNIQUE FOR DIELECTRIC MEASUREMENTS ON INDIVIDUAL CELLS AND PARTICLES. J. Electrostat. 1988, 21 (2-3), 151-191.

(3) Hughes, M. P.; Morgan, H. Dielectrophoretic Characterization and Separation of Antibody-Coated Submicrometer Latex Spheres. Anal. Chem. 1999, 71 (16), 3441-3445.

(4) Marszalek, P.; Zielinsky, J. J.; Fikus, M.; Tsong, T. Y. Determination of Electric Parameters of Cell Membranes by a Dielectrophoresis Method. Biophys. J. 1991, 59 (5), 982-987.

(5) Härdle, W. K.; Simar, L. Applied Multivariate Statistical Analysis; Dordrecht : Springer, 2012. 
(6) Haandbæk, N.; Bürgel, S. C.; Heer, F.; Hierlemann, A. Characterization of Subcellular Morphology of Single Yeast Cells Using High Frequency Microfluidic Impedance Cytometer. Lab Chip 2014, 14 (2), 369-377.

(7) Pauly, H.; Schwan, H. P. Über Die Impedanz Einer Suspension von Kugelförmigen Teilchen Mit Einer Schale. Zeitschrift für Naturforsch. B 1959, 14 (2), 125-131.

(8) Irimajiri, A.; Hanai, T.; Inouye, A. A Dielectric Theory of "multi-Stratified Shell" Model with Its Application to a Lymphoma Cell. J. Theor. Biol. 1979, 78 (2), 251-269.

(9) Gawad, S.; Cheung, K.; Seger, U.; Bertsch, A.; Renaud, P. Dielectric Spectroscopy in a Micromachined Flow Cytometer: Theoretical and Practical Considerations. Lab Chip 2004, 4 (3), 241-251.

(10) van den Boomgaard, R.; van Balen, R. Methods for Fast Morphological Image Transforms Using Bitmapped Binary Images. CVGIP Graph. Model. Image Process. 1992, 54 (3), 252-258.

(11) Murty, K. G. An Algorithm for Ranking All the Assignments in Order of Increasing Cost. Oper. Res. 1968, 16 (3), pp. 682687.

(12) Kalman, R. E. A New Approach to Linear Filtering and Prediction Problems. J. Basic Eng. 1960, 82 (1), 35. 